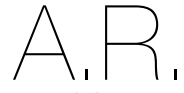

1 Escola Superior de Tecnologia da Saúde de Coimbra do Instituto Politécnico de Coimbra, Rua 5 de Outubro - SM Bispo, Apartado 7006, 3046-854 Coimbra, Portugal

*Endereço para correspondência:

Daniela Carvalho Urbanização da Maligueira, lote $9-5^{\circ} \mathrm{C}$

2415-341 Leiria, Portuga dani.romina@hotmail.com

Histórico do artigo:

Recebido a 28 de janeiro de 2020 Aceite a 30 de junho de 2020

\title{
MICROBIOTA INTESTINAL E OBESIDADE INFANTIL - UMA REVISÃO NARRATIVA
}

\author{
INTESTINAL MICROBIOTA AND CHID OBESITY - A REVIEW
}

Daniela Carvalho"*; Ana Faria'; Helena Loureiro'

\section{RESUMO}

INTRODUÇÃo: A composição da microbiota intestinal tem sido cada vez mais apontada como um fator preponderante para a obesidade, nomeadamente a obesidade infantil, uma vez que a infância é um período crítico para o desenvolvimento das bactérias colonizadoras do intestino.

OBJETIVOS: O objetivo deste trabalho é realizar uma revisão narrativa, de forma a verificar as diferenças na composição da microbiota intestinal das crianças e adolescentes com excesso de peso/obesidade comparativamente às normoponderais, através da análise de artigos científicos, de intervenção e de observação, com foco no papel da microbiota intestinal na idade pediátrica.

METODOLOGIA: Foi efetuada uma recolha e análise de artigos, sendo que foram selecionados 14 artigos para comporem esta revisão. RESULTADOS: O aumento do rácio firmicutes/bacteroidetes está associado ao aumento do peso/índice de massa corporal infantil. Verifica-se também que tanto a diversidade como a quantidade total de bactérias no intestino, variam de acordo com o peso corporal e que fatores como a dieta, suplementação em probióticos e antibióticos podem ter um papel preponderante na formação da microbiota intestinal infantil.

CONCLUSÕES: A informação analisada sugere que existe uma associação entre a composição da microbiota intestinal e o peso corporal. Contudo, ainda são poucos os estudos existentes para poder reconhecer de forma clara e precisa o papel da microbiota intestinal na etiologia da obesidade infantil.

\section{PALAVRAS-CHAVE}

Microbiota intestinal, Obesidade infantil

\section{ABSTRACT}

INTRODUCTION: The composition of the intestinal microbiota has been increasingly pointed as a major factor for obesity, particularly in childhood obesity since childhood is a critical period for the development of colonizing bacteria in the gut.

OBJECTIVES: The aim of this study is to carry out a narrative review, in order to verify differences in the composition of the intestinal microbiota of children and adolescents with overweight/obesity compared to normal weight, through the analysis of scientific articles, interventions and observations, focusing on the role of intestinal microbiota in pediatric age.

METHODOLOGY: A selection and analysis of articles was performed, and 14 articles were selected to compose this review. RESULTS: The group of bacteria most associated with weight gain / body mass index in children are bacteroidetes and firmicutes. It is also found that diversification and bacterial quantification of the intestinal microbiota vary according to body weight, and that factors such as diet, probiotic supplementation and antibiotics may have a major role in the formation of childhood intestinal microbiota. CoNCLusions: The analyzed information suggests that there is an association between intestinal microbiota composition and body weight. However, there are still few studies to be able to clearly and precisely recognize the role of intestinal microbiota in the etiology of childhood obesity.

\section{KEYWORDS}

Intestinal Microbiota, Childhood obesity

\section{INTRODUÇÃO}

A obesidade é atualmente considerada uma das maiores problemáticas da saúde pública, estando associada ao aparecimento de múltiplas patologias (1). Um fator bastante preocupante é o aumento da obesidade infantil, fator de risco para obesidade em idade adulta e, que tem aumentado nas últimas décadas (1).

Mais recentemente, algumas investigações clínicas têm apontado para diferenças significativas que ocorrem na composição da microbiota intestinal em crianças obesas e não obesas. A microbiota intestinal é o conjunto de microrganismos que habitam o trato gastrointestinal e que influenciam o balanço energético, produzindo ácidos gordos de cadeia curta (AGCC) a partir da digestão de polissacarídeos (1, 2). A composição dessa comunidade microbiana depende do hospedeiro, mas também pode 
ser modificada por fatores exógenos e endógenos. No que diz respeito ao hospedeiro, essas bactérias são simbióticas e desempenham um papel importante nos processos fisiológicos (ex.: digestão), ou podem intervir no metabolismo (podem aumentar a produção de energia a partir da dieta). Todos esses processos estão envolvidos na obesidade e distúrbios metabólicos (1).

A infância é um período crítico no desenvolvimento das bactérias colonizadoras do intestino, com um aumento gradual da colonização de Bacteroidetes a partir do momento do nascimento (3). A composição da microbiota intestinal é definida nesta altura e evolui até aos 3 anos de idade, permanecendo constante durante a vida, sendo que alguns fatores podem alterá-la temporariamente, como os antibióticos e a alimentação (2).

A maioria das bactérias presentes no intestino pertence a um dos seguintes filos: Bacteroidetes, Firmicutes, Actinobacteria, Proteobacteria e Verrucomicrobia. Os principais filos são os Firmicutes e os Bacteroidetes aos quais pertencem $90 \%$ das bactérias do intestino.

A composição da diversidade bacteriana aparenta mudar entre eutotróficos e obesos (1). Desta forma, o objetivo deste trabalho é realizar uma revisão acerca da associação entre a microbiota intestinal e o peso corporal.

\section{METODOLOGIA}

Foi realizada uma revisão da literatura através da base de dados Pubmed. O limite temporal para a pesquisa dos artigos científicos foi de 10 anos (artigos publicados desde abril de 2009 até abril 2019).
Tendo em conta o objetivo desta revisão, de forma a aumentar a eficiência da pesquisa e obter todos os sinónimos reunidos num só descritor, a pesquisa foi realizada recorrendo ao MeSH. Desta forma as palavras-chaves usadas foram: "Gastrointestinal Microbiome" e "Pediatric Obesity", recorrendo ao operador booleano AND. No entanto, tendo em conta que artigos mais recentes que dão entrada na Pubmed podem ainda não ter termos MeSH atribuídos, foi realizada pesquisa (com limite temporal "último mês") com as seguintes palavras chaves: "gut microbiota", "gastrointestinal microbiota", "intestinal microbiota", "gut microbiome", "gastrointestinal microbiome", "intestinal microbiome", "child obesity", "infant obesity", "adolescente obesity" e, para facilitar a pesquisa foram usados operadores lógicos booleanos como a truncatura $\left({ }^{*}\right)$ e o AND.

\section{RESULTADOS}

No total foram encontrados 38 artigos, sem duplicados, dos quais foram excluídos 24 por serem artigos de revisão e/ou artigos cujo objetivo não era a relação entre a microbiota intestinal e a obesidade infantil. Em suma, no final da pesquisa foram selecionados 14 artigos para compor esta revisão. A Tabela 1 apresenta uma síntese dos estudos incluído nesta revisão.

Dos 14 estudos, 3 foram de intervenção e os restantes de observação. Verifica-se que tanto a diversidade como a quantidade total de bactérias no intestino variam de acordo com o peso corporal e que fatores como a dieta, suplementação (probióticos) e antibióticos podem ter um papel na formação da microbiota intestinal infantil.

\section{Tabela 1}

Síntese dos estudos realizados no âmbito da temática "Microbiota intestinal - Obesidade infantil"

\begin{tabular}{|c|c|c|c|c|c|}
\hline $\begin{array}{c}\text { ESTUDO } \\
\text { (AUTORES/ANO) }\end{array}$ & AMOSTRA & OBJETIVO & METODOLOGIA & $\begin{array}{l}\text { RESULTADOS/EVIDÊNCIAS } \\
\text { MAIS SIGNIFICATIVAS }\end{array}$ & LIMITAÇÕES \\
\hline $\begin{array}{l}\text { Santacruz et } \\
\text { al (2009) (4) }\end{array}$ & $\begin{array}{l}36 \text { crianças dos } \\
13 \text { aos } 15 \text { anos }\end{array}$ & $\begin{array}{l}\text { Determinar a influência de um } \\
\text { programa multidisciplinar no } \\
\text { tratamento da obesidade na } \\
\text { microbiota intestinal e peso de } \\
\text { adolescentes com excesso de } \\
\text { peso/obesidade. }\end{array}$ & $\begin{array}{l}\text { - Peso, altura, pregas e diário alimentar } \\
\text { ( } 72 \text { horas) antes e após a intervenção - } \\
10 \text { semanas de restrição calórica de } \\
\text { acordo com nível de obesidade ( } 10 \text { a } \\
40 \% \text { ) e aumento da atividade física; } \\
\text { - Grupos bacterianos da microbiota } \\
\text { intestinal analisados antes e após a } \\
\text { intervenção. }\end{array}$ & $\begin{array}{l}\text { - Grupo com maior restrição } \\
\text { calórica }(\mathrm{n}=23 \text { ) perdeu }>4,0 \mathrm{~kg} \\
\text { após a intervenção (restantes } \\
\text { perderam <2,0 kg) - aumento das } \\
\text { contagens do grupo Bacteroides } \\
\text { fragilis e Lactobacillus, e diminuição } \\
\text { de Clostridium coccoides, } \\
\text { Bifidobacterium longum e } \\
\text { Bifidobacterium adolescentis. }\end{array}$ & $\begin{array}{l}\text { - Avaliação dietética } \\
\text { limitada } \\
\text { - Amostra muito pequena }\end{array}$ \\
\hline $\begin{array}{l}\text { Balamurugan } \\
\text { et al (2009) } \\
\text { (14) }\end{array}$ & $\begin{array}{l}28 \text { crianças dos } \\
11 \text { aos } 14 \text { anos }\end{array}$ & $\begin{array}{l}\text { Examinar a composição } \\
\text { predominante na micobiota } \\
\text { fecal em crianças obesas e não } \\
\text { obesas. }\end{array}$ & $\begin{array}{l}\text { - } 15 \text { crianças obesas e } 13 \\
\text { normoponderais - questionário } \\
\text { alimentar das } 24 \text { horas anteriores, } \\
\text { questionário de frequência alimentar } \\
\text { (QFA) (últimos } 3 \text { meses) e amostras } \\
\text { fecais. }\end{array}$ & $\begin{array}{l}\text { - Ingestão de energia e } \\
\text { macronutrientes semelhante nas } \\
\text { crianças obesas e normoponderais; } \\
\text { - Níveis de Faecalibacterium } \\
\text { prausnitzii significativamente } \\
\text { maiores nas crianças obesas. }\end{array}$ & - Amostra muito pequena \\
\hline $\begin{array}{l}\text { Karlsson et al } \\
(2012)(15)\end{array}$ & $\begin{array}{c}40 \text { crianças dos } \\
4 \text { aos } 5 \text { anos } \\
\text { (15) }\end{array}$ & $\begin{array}{l}\text { Investigar a microbiota intestinal } \\
\text { em crianças do pré-escolar } \\
\text { com e sem excesso de peso/ } \\
\text { obesidade. }\end{array}$ & $\begin{array}{l}\text { - Gl: } 20 \text { crianças acima do peso } \\
\text { selecionados aleatoriamente antes do } \\
\text { início do programa e GC: } 20 \text { crianças } \\
\text { com o IMC normoponderal; } \\
\text { - Amostras fecais do GC e do Gl e, } \\
\text { amostras de sangue do Gl. }\end{array}$ & $\begin{array}{l}\text { - Concentração de } \\
\text { Enterobacteriaceae } \\
\text { significativamente maior nas } \\
\text { crianças obesas/excesso peso; } \\
\text { - Diminuição da diversidade } \\
\text { bacteriana em crianças com } \\
\text { excesso de peso. }\end{array}$ & $\begin{array}{l}\text { - Amostra muito pequena } \\
\text { - Não foram obtidas } \\
\text { amostras de sangue } \\
\text { do GC }\end{array}$ \\
\hline $\begin{array}{l}\text { Xu et al (2012) } \\
\text { (16) }\end{array}$ & $\begin{array}{l}175 \text { crianças (de } \\
14 \text { escolas) dos } \\
7 \text { aos } 13 \text { anos }\end{array}$ & $\begin{array}{l}\text { Investigar uma possível } \\
\text { correlação entre a microbiota } \\
\text { intestinal, Bacteroidetes e } \\
\text { Firmicutes, e a obesidade em } \\
\text { crianças. }\end{array}$ & $\begin{array}{l}\text { - Análise de amostras fecais } \\
\text { (determinação do número de } \\
\text { Bacteroidetes e Firmicutes na } \\
\text { microbiota intestinal) e de sangue } \\
\text { em jejum; } \\
\text { - Obtido peso e altura das crianças } \\
\text { (e IMC). }\end{array}$ & $\begin{array}{l}\text { - } 84 \text { crianças diagnosticadas } \\
\text { com excesso peso/obesidade e } \\
91 \text { com peso normal; } \\
\text { - O número de Bacteroides } \\
\text { e a relação Bact/Firm foi } \\
\text { significativamente menor no grupo } \\
\text { de obesos (diferença significativa } \\
\text { apenas nas meninas). }\end{array}$ & - Amostra pequena \\
\hline $\begin{array}{l}\text { Bervoets et al } \\
\text { (2013) (5) }\end{array}$ & $\begin{array}{c}53 \text { crianças dos } \\
6 \text { aos } 16 \text { anos }\end{array}$ & $\begin{array}{l}\text { Investigar e comparar a } \\
\text { composição da microbiota } \\
\text { intestinal em crianças obesas e } \\
\text { normoponderais. }\end{array}$ & $\begin{array}{l}\text { - } 26 \text { crianças obesas e } 27 \\
\text { normoponderais - amostras fecais; } \\
\text { - Parâmetros bioquímicos analisados } \\
\text { nas crianças com excesso de peso } \\
\text { e obesidade. }\end{array}$ & $\begin{array}{l}\text { - Crianças obesas apresentaram } \\
\text { uma relação elevada entre } \\
\text { Firmicutes e Bacteroidetes. } \\
\text { Baixas proporções relativas de } B \text {. } \\
\text { vulgatus e altas concentrações } \\
\text { de Lactobacillus observadas na } \\
\text { microbiota obesa. }\end{array}$ & $\begin{array}{l}\text { - Amostra pequena } \\
\text { - Fatores relacionados } \\
\text { à gravidez e estado } \\
\text { socioeconómico não } \\
\text { considerados }\end{array}$ \\
\hline
\end{tabular}


Tabela 1

Síntese dos estudos realizados no âmbito da temática "Microbiota intestinal - Obesidade infantil" - Continuação

\begin{tabular}{|c|c|c|c|c|c|}
\hline $\begin{array}{l}\text { Gao et al } \\
\text { (2015) (11) }\end{array}$ & $\begin{array}{c}126 \text { crianças: } \\
63 \text { (Gl) e } 63 \text { (GC) }\end{array}$ & $\begin{array}{l}\text { Determinar a correlação } \\
\text { entre a obesidade infantil e o } \\
\text { desequilíbrio da microbiota } \\
\text { intestinal, em crianças obesas } \\
\text { em comparação com as não } \\
\text { obesas. }\end{array}$ & $\begin{array}{l}\text { - Gl (crianças obesas) e GC (crianças } \\
\text { normoponderais) - emparelhadas de } \\
\text { acordo com idade e sexo. Registado } \\
\text { peso e altura; } \\
\text { - Amostras fecais (para determinar o } \\
\text { número de cópias de Bifidobacteria e } \\
\text { E.coli - a razão B/E foi calculada } \\
\text { e analisada estatisticamente). }\end{array}$ & $\begin{array}{l}\text { - Gl (crianças obesas) têm menor } \\
\text { quantidade de Bifidobactérias e } \\
\text { maior quantidade de } E \text {. coli (menor } \\
\text { relação B / E) em comparação com } \\
\text { o GC. }\end{array}$ & - Amostra pequena \\
\hline $\begin{array}{l}\text { Contreras et al } \\
\text { (2017) (6) }\end{array}$ & $\begin{array}{l}138 \text { crianças } \\
\text { dos } 6 \text { aos } 12 \\
\text { anos }\end{array}$ & $\begin{array}{l}\text { Avaliar as diferenças na } \\
\text { composição da microbiota } \\
\text { intestinal de crianças mexicanas } \\
\text { em idade escolar obesas e } \\
\text { normoponderais. }\end{array}$ & $\begin{array}{l}\text { - } 71 \text { crianças obesas e } 67 \\
\text { normoponderais - parâmetros } \\
\text { antropométricos, pressão arterial e \% } \\
\text { de gordura corporal (\%GC) avaliados; } \\
\text { - QFA semiquantitativo (respondido } \\
\text { pelos pais); } \\
\text { - Amostras fecais e análises bioquímicas } \\
\text { realizadas. }\end{array}$ & $\begin{array}{l}\text { - A abundância de Bacteroides } \\
\text { eggerthii foi significativamente maior } \\
\text { em crianças obesas e correlacionou- } \\
\text { se positivamente com a \%GC e } \\
\text { negativamente } \\
\text { com o consumo de fibra insolúvel. }\end{array}$ & $\begin{array}{l}\text { - Amostra pequena } \\
\text { - Apenas } 65 \text { crianças } \\
\text { responderam ao QFA }\end{array}$ \\
\hline $\begin{array}{l}\text { Riva et al } \\
(2017)(8)\end{array}$ & $\begin{array}{c}78 \text { crianças dos } \\
6 \text { aos } 16 \text { anos }\end{array}$ & $\begin{array}{l}\text { Caraterizar a composição da } \\
\text { microbiota intestinal em crianças } \\
\text { obesas e normoponderais. }\end{array}$ & $\begin{array}{l}\text { - } 42 \text { crianças obesas e } 36 \\
\text { normoponderais - amostras fecais } \\
\text { (comparação dos perfis da microbiota } \\
\text { intestinal com os AGCC e z-scores de } \\
\text { IMC) e dados dos hábitos alimentares } \\
\text { obtidos; } \\
\text { - Características como idade, sexo, } \\
\text { IMC, modo de parto e história de } \\
\text { amamentação - consideradas. }\end{array}$ & $\begin{array}{l}\text { - A obesidade foi associada a uma } \\
\text { microbiota intestinal alterada por } \\
\text { níveis elevados de Firmicutes; } \\
\text { - Os AGCC, foram mais elevados } \\
\text { nas crianças obesas, reforçando a } \\
\text { ligação entre a microbiota, os AGCC } \\
\text { e a obesidade. }\end{array}$ & - Amostra pequena \\
\hline $\begin{array}{l}\text { Hou et al } \\
\text { (2017) (12) }\end{array}$ & $\begin{array}{c}143 \text { crianças } \\
\text { dos } 3 \text { aos } \\
18 \text { anos: } 87 \\
\text { obesas (Gl) e } 56 \\
\text { normoponderais } \\
\text { (GC) }\end{array}$ & $\begin{array}{l}\text { Investigar as diferenças na } \\
\text { microbiota intestinal entre } \\
\text { crianças obesas e não obesas. }\end{array}$ & $\begin{array}{l}\text { - } 11 \text { das crianças obesas (Gl) receberam } \\
\text { fórmula dietética usada num estudo } \\
\text { anterior - foram reamostradas } 30,60 \\
\text { e } 90 \text { dias após a intervenção; } \\
\text { - Nenhuma das coortes tomou } \\
\text { antibiótico nos } 3 \text { meses anteriores } \\
\text { à amostragem; } \\
\text { - Amostras fecais (comparação da } \\
\text { estrutura geral da microbiota fecal } \\
\text { do Gl com o GC). }\end{array}$ & $\begin{array}{l}\text { - Firmicutes e Bacteroidetes foram } \\
\text { o microbioma fecal predominante } \\
\text { em ambas as coortes. No entanto, } \\
\text { a relação de abundância relativa de } \\
\text { Firmicutes e Bacteroidetes (F/B) na } \\
\text { coorte obesa foi significativamente } \\
\text { maior; } \\
\text { - Bifidobacterium e Lactobacillus } \\
\text { aumentaram no Gl (durante o } \\
\text { processo de redução de peso). }\end{array}$ & - Amostra pequena \\
\hline $\begin{array}{l}\text { Bai et al (2018) } \\
\text { (10) }\end{array}$ & $\begin{array}{l}267 \text { crianças } \\
\text { dos } 7 \text { aos } 18 \\
\text { anos (american } \\
\text { gut project) }\end{array}$ & $\begin{array}{l}\text { Investigar as associações entre } \\
\text { a microbiota intestinal, o IMC } \\
\text { e estilos de vida (tipo de dieta } \\
\text { e frequência de exercício), } \\
\text { controlando fatores demográficos } \\
\text { e clínicos entre crianças dos } 7 \\
\text { aos } 18 \text { anos. }\end{array}$ & $\begin{array}{l}\text { - Estudo de coorte - informações } \\
\text { como idade, sexo, raça, altura/peso } \\
\text { e IMC obtidas. Idade das crianças foi } \\
\text { estratificada em } 2 \text { grupos (crianças dos } \\
7 \text { aos } 12 \text { anos de idade vs adolescentes } \\
\text { dos } 13 \text { aos } 17 \text { anos); } \\
\text { - Composição da microbiota intestinal e } \\
\text { associações com o IMC e estilos de vida } \\
\text { analisadas; } \\
\text { - Variáveis como o uso de antibióticos e/ } \\
\text { ou probióticos foi tido em consideração; } \\
\text { - Cada criança forneceu respostas num } \\
\text { questionário sobre o tipo de dieta e } \\
\text { frequência de exercício físico. }\end{array}$ & $\begin{array}{l}\text { - O uso de antibióticos e probióticos } \\
\text { afetou significativamente a } \\
\text { diversidade da microbiota } \\
\text { intestinal (diminuiu e aumentou } \\
\text { respetivamente); } \\
\text { - A microbiota intestinal foi } \\
\text { dominada pelos filos bacterianos } \\
\text { Firmicutes, Bacteroidetes e } \\
\text { Proteobacteria; } \\
\text { - Lactobacillus e Bifidobacterium } \\
\text { foram associados com um maior } \\
\text { nível de IMC. }\end{array}$ & $\begin{array}{l}\text { - Participantes eram } \\
\text { predominantemente } \\
\text { caucasianos não } \\
\text { hispânicos }(85 \%)\end{array}$ \\
\hline $\begin{array}{l}\text { Hollister et al } \\
\text { (2018) (17) }\end{array}$ & $\begin{array}{l}52 \text { crianças dos } \\
2 \text { aos } 5 \text { anos: } 30 \\
\text { obesas (Gl) e } 22 \\
\text { normoponderais } \\
\text { (GC) }\end{array}$ & $\begin{array}{l}\text { Avaliar associações entre o peso } \\
\text { corporal e a microbiota intestinal } \\
\text { ao longo do tempo em crianças } \\
\text { obesas em idade pré-escolar. }\end{array}$ & $\begin{array}{l}\text { - Ensaio clínico randomizado de base } \\
\text { comportamental - crianças obesas } \\
\text { hispânicas em idade pré-escolar } \\
\text { (duração de } 6 \text { meses); } \\
\text { - GC constituído por crianças da mesma } \\
\text { faixa etárias e etnia, mas com peso } \\
\text { normal; } \\
\text { - Peso, altura (z scores IMC calculados) } \\
\text { e dados dietéticos recolhidos (início e } \\
\text { seguimento do estudo); } \\
\text { - Amostras fecais (início e fim do estudo) } \\
\text { - razão Firmicutes/Bacteroidetes (F/B) } \\
\text { determinada. }\end{array}$ & $\begin{array}{l}\text { - Pequenas diferenças o Gl e o GC } \\
\text { no que diz respeito à composição da } \\
\text { microbiota, mas sem diferenças em } \\
\text { relação à F/B; } \\
\text { - Das } 30 \text { crianças obesas (Gl) entre } \\
\text { a pré-intervenção e pós-intervenção, } \\
\text { foi observada uma diminuição do } \\
\text { IMC; } \\
\text { - Consumo de açúcar diminuiu de } \\
\text { uma média de } 54 \mathrm{~g} / \text { dia para } 46 \mathrm{~g} / \mathrm{dia} \\
\text { (em ambos os grupos). }\end{array}$ & $\begin{array}{l}\text { - Amostra pequena } \\
\text { - Não foram recolhidos } \\
\text { alguns dados do GC (ex.: } \\
\text { IMC parental), pelo que } \\
\text { variáveis potencialmente } \\
\text { não-confiáveis podem ser } \\
\text { uma limitação. }\end{array}$ \\
\hline
\end{tabular}

- Microbiota intestinal avaliada em 6

pontos de tempo nos primeiros 2 anos de vida;

- Altura e peso aferidos aos 12 anos;

- IMC pré-gestacional (autorrelato

$\begin{array}{ll}\text { Examinar a microbiota intestinal } & \cdot \text { IMC pre-gestacional (autorrelato } \\ \text { materno) e ganho de peso gestacional }\end{array}$

Stanislawski

materno) e ganho de peso gestaciona
excessivo calculado;

et al (2018) (9)

165 crianças nos primeiros 2 anos de vida e

a sua relação com de idade.

- Questionários maternos $(1,6,12$

24 meses) - modo de parto, idade
materna, educação, tabagismo materno,

etnia, sexo infantil, uso de antibióticos,

práticas de amamentação e introdução

de alimentos.

- Aos 2/3 anos de idade, peso e CC

avaliados. Obtido valor da massa isenta

de gordura (MIG), MG e IMC;

Smith-Brown Explorar a associação entre

et al (2018) 37 crianças dos o perfil da microbiota, peso

- Modo de parto (normal ou cesariana),

nível de escolaridade da mãe e uso de

antibióticos nos 6 meses anteriores

foram registados;

crianças pequenas.

- Amostras fecais obtidas e analisadas.

- A microbiota intestinal durante

os primeiros 2 anos de vida

(particularmente aos 2 anos de

idade), fortemente associada a um

IMC tardio na infância;

- Níveis elevados de firmicutes nas

crianças com excesso de peso/

obesidade.
- Amostra pequena

- Perfil de microbiota fecal foi

significativamente associado ao tamanho corporal e composição

corporal (análises sexuais

específicas revelaram associação

mais significativa entre a

composição da microbiota e a MIG

nos meninos). 


\begin{tabular}{|c|c|c|c|c|c|}
\hline $\begin{array}{l}\text { Nirmalkar et al } \\
(2018)(7)\end{array}$ & $\begin{array}{l}172 \text { crianças } \\
\text { (6 aos } 11 \text { anos }) \\
\text { e adolescentes } \\
\text { (12 aos } 18 \text { anos) }\end{array}$ & $\begin{array}{l}\text { Estudar a associação entre } \\
\text { a diversidade da microbiota } \\
\text { intestinal e os marcadores de } \\
\text { disfunção endotelial em crianças } \\
\text { e adolescentes mexicanas } \\
\text { obesas. }\end{array}$ & $\begin{array}{l}\text { - Seleção da amostra por pediatra em } \\
\text { entrevista (saudáveis, sem doenças } \\
\text { gastrointestinais ou uso de probióticos } \\
\text { nos últimos } 3 \text { meses) - } 111 \text { crianças e } \\
61 \text { adolescentes divididos em } 2 \text { grupos: } \\
\text { peso normal ( } n=49 \text { ) e obeso }(n=62) \text { em } \\
\text { crianças e peso normal }(n=27) \text { e obeso } \\
(n=34) \text { em adolescentes; } \\
\text { - Peso/altura, CC e ingestão alimentar } \\
\text { (diário alimentar de } 7 \text { dias - nutricionista) } \\
\text { avaliados; } \\
\text { - Amostras de sangue e amostras fecais } \\
\text { obtidas. }\end{array}$ & $\begin{array}{l}\text { - Crianças e adolescentes obesos } \\
\text { apresentaram níveis mais elevados } \\
\text { de resistência à insulina, síndrome } \\
\text { metabólica, hipertrigliceridemia e } \\
\text { hipercolesterolemia); } \\
\text { - Crianças e adolescentes obesos } \\
\text { tiveram níveis aumentados de } \\
\text { proteína C -reativa e uma redução } \\
\text { da adiponectina, afetando a função } \\
\text { endotelial e associando-se a } \\
\text { alterações na microbiota intestinal } \\
\text { (aumento de Bacteroidales e } \\
\text { Lactobacillis). }\end{array}$ & $\begin{array}{l}\text { - Amostra pequena e } \\
\text { homogénea }\end{array}$ \\
\hline
\end{tabular}

AGCC: Ácidos gordos de cadeia curta

CC: Circunferência da cintura

GC: Grupo de controlo

Gl: Grupo de intervenção

\section{ANÁLISE CRÍTICA}

A necessidade de clarificação do papel da microbiota intestinal no ganho de peso, nomeadamente desde a infância, tem motivado a realização de diferentes estudos nos últimos anos.

Nesta revisão, verificou-se que o grupo de bactérias mais associado ao aumento do peso/IMC infantil são os bacteroidetes (4-7) e os firmicutes (5, 7-9).

A presença de Lactobacillus foi associada a um maior IMC $(5,7$, 10), exceto em um estudo (4), enquanto que a de Staphylococcus foi relacionado a um menor IMC (5). Quanto às Bifidobacterias, microorganismos reconhecidos pelo seu papel benéfico na microbiota intestinal, nos estudos que quantificaram este grupo, verificou-se um baixo nível nas crianças com excesso de peso/obesidade (11) (no entanto, num estudo (4) no processo de perda de peso houve uma diminuição deste grupo) e um aumento no estudo de Hou et al (12), no processo de perda de peso.

Alguns fatores de risco considerados para ganho de peso têm sido considerados. A suplementação com probióticos (10) demonstrou uma associação positiva entre o número de Bifidobactérias e peso corporal. Outro fator de risco considerado foi a toma de antibióticos que, segundo o estudo de Bai et al (10) diminui a diversidade bacteriana da microbiota intestinal.

Embora esta revisão forneça informações úteis sobre a microbiota intestinal e a obesidade na população pediátrica, como, por exemplo, verificar-se que a composição da microbiota intestinal difere de acordo com o peso corporal (crianças normoponderais versus crianças com excesso de peso/obesidade), são ainda poucos os estudos existentes nesta temática com uma amostra significativa para poder reconhecer de forma clara e precisa o papel da microbiota intestinal na etiologia da obesidade infantil.

\section{CONCLUSÕES}

Parece existir uma associação entre a composição da microbiota intestinal e o peso corporal e, que os filos bacterianos mais estudados e com maior evidência de associação com o excesso de peso/ obesidade são os Firmicutes e os Bacteroidetes.

No entanto, dado o limitado número de publicações que avaliam esses filos completos e com amostras significativas, serão necessários mais estudos para compreender de forma precisa a relação entre a microbiota intestinal e a obesidade e mais ensaios clínicos de caráter intervencional.

\section{REFERÊNCIAS BIBLIOGRÁFICAS}

1. Castaner O, Goday A, Park YM, Lee SH, Magkos F, Shiow SATE, et al. The gut microbiome profile in obesity: A systematic review. Int J Endocrinol. 2018;2018. 2. Oliveira RCS, Coelho PMB de S, Estevan M del CL. Does microbiota influence the risk of childhood obesity? Rev Esp Nutr Humana y Diet. 2018;22(2):157-68.

3. Koleva PT, Bridgman SL, Kozyrskyj AL. The infant gut microbiome: Evidence for
IMC: Índice de massa corporal

MG: Massa gorda

MIG: Massa isenta de gordura

QFA: Questionário de frequência alimentar

obesity risk and dietary intervention. Nutrients. 2015;7(4):2237-60

4. Santacruz A, Marcos A, Wärnberg J, Martí A, Martin-Matillas M, Campoy C, et al. Interplay between weight loss and gut microbiota composition in overweight adolescents. Obesity [Internet]. 2009;17(10):1906-15. Available from: http://dx.doi.org/10.1038/oby.2009.112. 5. Bervoets L, Van Hoorenbeeck K, Kortleven I, Van Noten C, Hens N, Vael C, et al. Differences in gut microbiota composition between obese and lean children: A crosssectional study. Gut Pathog. 2013;5(1):1-10.

6. López-Contreras BE, Morán-Ramos S, Villarruel-Vázquez R, Macías-Kauffer L, Villamil-Ramírez H, León-Mimila P, et al. Composition of gut microbiota in obese and normal-weight Mexican school-age children and its association with metabolic traits. Pediatr Obes. 2018;13(6):381-8.

7. Nirmalkar K, Murugesan S, Pizano-Zárate ML, Villalobos-Flores LE, García-González C, Morales-Hernández RM, et al. Gut microbiota and endothelial dysfunction markers in obese Mexican children and adolescents. Nutrients. 2018;10(12).

8. Riva A, Borgo F, Lassandro C, Verduci E, Morace G, Borghi E, et al. Pediatric obesity is associated with an altered gut microbiota and discordant shifts in Firmicutes populations. Environ Microbiol. 2017;19(1):95-105.

9. Stanislawski MA, Dabelea D, Wagner BD, Iszatt N, Dahl C, Sontag MK, et al. Gut Microbiota in the First 2 Years of Life and the Association with Body Mass Index at Age 12 in a Norwegian Birth Cohort. MBio. 2018;9(5):1-14.

10. Bai J, Hu Y, Bruner DW. Composition of gut microbiota and its association with body mass index and lifestyle factors in a cohort of 7-18 years old children from the American Gut Project. Pediatr Obes. 2019;14(4).

11. Gao X, Jia R, Xie L, Kuang L, Feng L, Wan C. Obesity in school-aged children and its correlation with Gut E.coli and Bifidobacteria: A case-control study. BMC Pediatr [Internet]. 2015;15(1):1-4.

12. Hou YP, He QQ, Ouyang HM, Peng HS, Wang Q, Li J, et al. Human Gut Microbiota Associated with Obesity in Chinese Children and Adolescents. Biomed Res Int. 2017;2017.

13. Smith-Brown P, Morrison M, Krause L, Davies PSW. Male-specific association between fat-free mass index and fecal microbiota in 2- to 3-year-old Australian children. J Pediatr Gastroenterol Nutr. 2018;66(1):147-51.

14. Balamurugan R, George G, Kabeerdoss J, Hepsiba J, Chandragunasekaran AMS, Ramakrishna BS. Quantitative differences in intestinal Faecalibacterium prausnitzii in obese Indian children. Br J Nutr. 2010;103(3):335-8.

15. Karlsson CLJ, Önnerfält J, Xu J, Molin G, Ahrné S, Thorngren-Jerneck K. The microbiota of the gut in preschool children with normal and excessive body weight. Obesity. 2012;20(11):2257-61.

16. Xu P, Li M, Zhang J, Zhang T. Correlation of intestinal microbiota with overweight and obesity in Kazakh school children. BMC Microbiol. 2012;12.

17. Hollister EB, Foster BA, Dahdouli M, Ramirez J, Lai Z. Characterization of the Stool Microbiome in Hispanic Preschool Children by Weight Status and Time. Child Obes. 2018;14(2):122-30. 